\title{
A rapid and accurate approach for prediction of interactomes from co-elution data (PrInCE)
}

\author{
R. Greg Stacey ${ }^{1 *}$ (D), Michael A. Skinnider ${ }^{1}$, Nichollas E. Scott ${ }^{1,2}$ and Leonard J. Foster ${ }^{1,3^{*}}$
}

\begin{abstract}
Background: An organism's protein interactome, or complete network of protein-protein interactions, defines the protein complexes that drive cellular processes. Techniques for studying protein complexes have traditionally applied targeted strategies such as yeast two-hybrid or affinity purification-mass spectrometry to assess protein interactions. However, given the vast number of protein complexes, more scalable methods are necessary to accelerate interaction discovery and to construct whole interactomes. We recently developed a complementary technique based on the use of protein correlation profiling (PCP) and stable isotope labeling in amino acids in cell culture (SILAC) to assess chromatographic co-elution as evidence of interacting proteins. Importantly, PCP-SILAC is also capable of measuring protein interactions simultaneously under multiple biological conditions, allowing the detection of treatment-specific changes to an interactome. Given the uniqueness and high dimensionality of coelution data, new tools are needed to compare protein elution profiles, control false discovery rates, and construct an accurate interactome.

Results: Here we describe a freely available bioinformatics pipeline, PrlnCE, for the analysis of co-elution data. PrInCE is a modular, open-source library that is computationally inexpensive, able to use label and label-free data, and capable of detecting tens of thousands of protein-protein interactions. Using a machine learning approach, PrInCE offers greatly reduced run time, more predicted interactions at the same stringency, prediction of protein complexes, and greater ease of use over previous bioinformatics tools for co-elution data. PrInCE is implemented in Matlab (version R2017a). Source code and standalone executable programs for Windows and Mac OSX are available at https://github.com/fosterlab/PrlnCE, where usage instructions can be found. An example dataset and output are also provided for testing purposes.

Conclusions: PrInCE is the first fast and easy-to-use data analysis pipeline that predicts interactomes and protein complexes from co-elution data. PrInCE allows researchers without bioinformatics expertise to analyze highthroughput co-elution datasets.
\end{abstract}

Keywords: Interactome, Protein-protein interaction, Co-fractionation, Co-elution, Protein correlation profiling, Proteomics, System biology, Data analysis, Software

\footnotetext{
*Correspondence: richard.greg.stacey@ubc.msl.ca; foster@msl.ubc.ca

${ }^{1}$ Michael Smith Laboratories, University of British Columbia, Vancouver V6T

1Z4, Canada

Full list of author information is available at the end of the article
} International License (http://creativecommons.org/licenses/by/4.0/), which permits unrestricted use, distribution, and reproduction in any medium, provided you give appropriate credit to the original author(s) and the source, provide a link to the Creative Commons license, and indicate if changes were made. The Creative Commons Public Domain Dedication waiver (http://creativecommons.org/publicdomain/zero/1.0/) applies to the data made available in this article, unless otherwise stated. 


\section{Background}

The association of proteins into complexes is common across all domains of life $[1,2]$. Indeed, most proteins in well-studied proteomes are involved in at least one protein complex [3, 4]. Therefore, understanding the roles, mechanisms, and interplay of protein complexes is central to understanding life.

A proteome of 1500 proteins has over one million possible binary protein-protein interactions (PPIs) and many more potential higher-order complexes. Because of this combinatorial explosion, even relatively simple proteomes can yield rich, complex interactomes. High-throughput or high-content methods that identify many PPIs simultaneously are therefore valuable to efficiently map these networks. There are currently three general methods for doing this: The first, yeast-2 hybrid ( $\mathrm{Y} 2 \mathrm{H})$, operates by incorporating modified bait and prey proteins in a genetically modified yeast cell, such that a PPI between bait and prey drives transcription of a reporter gene. Affinity purification mass spectrometry (AP-MS), a second technique, involves immunoprecipitation of proteins of interest (baits) [5]. While powerful, both techniques face limitations. For one, tagging proteins, typically with Gal4 in the case of $\mathrm{Y} 2 \mathrm{H}$ or an epitope-antibody combination for AP-MS, creates nonendogenous conditions that can disrupt protein binding sites and increase the number of false negatives.

The third general approach, collectively termed cofractionation approaches, involves resolving complexes by either chromatography or electrophoresis and assigning interacting partners based on the similarity of fractionation profiles [6-8]. While there are similarities in how the data from these methods are treated, there are also unique considerations for each one. Being more established methods, $\mathrm{Y} 2 \mathrm{H}$ and AP-MS have several excellent approaches for data analysis $[5,9,10]$. However, there does not yet exist a gold standard tool for analyzing co-fractionation data. We [11] and others have previously reported pipelines for analyzing co-fractionation data, although existing approaches use other external sources of data, e.g. coevolution, in addition to co-fractionation data $[6,12]$. Optimally though, an interactome should be derived from cofractionation data alone, using other data only for benchmarking. To this end, here we describe an open-source pipeline for analyzing co-fractionation data: PrInCE (Prediction of Interactomes from Co-Elution). PrInCE represents a major conceptual advance over preliminary bioinformatics treatments published by our lab, which provided basic data extraction and curve fitting tools for co-elution data $[8,11]$. Improvements include ranked interactions, improved user interface, and extensive documentation. Importantly, PrInCE uses machine learning methods which greatly improve its performance. We benchmarked the performance of PrInCE versus a previous version [11] and demonstrate a 1.5-to-2-fold improvement in the number of predicted PPIs at a given false disovery rate with a $97 \%$ decrease in computational cost. This pipeline is freely available for download [13].

\section{Methods \\ Pipeline overview}

The workflow of the pipeline is divided into five modules: 1) identification of Gaussian-like peaks in the co-fractionation profiles (GaussBuild.m); 2) correction for slight differences in the separation dimension between replicates (Alignment.m); 3) comparison of differences in protein amounts, i.e. fold changes, between conditions (FoldChange.m); 4) prediction of PPIs within each condition (Interactions.m); and 5) construction of protein complexes from the predicted PPIs (Complexes.m). The first two modules, i.e. GaussBuild.m and Alignment.m, are pre-processing steps, while the remaining three modules compute protein abundance changes and predict protein interactions and complexes (Fig. 1).

\section{Requirements \\ Software and hardware}

PrInCE is available as a standalone program for Windows or Mac OSX, as well as a Matlab package. Matlab is not required to run standalone versions of PrInCE but it was selected initially due to superior curve fitting tools compared to other environments. After downloading and saving to a dedicated folder containing co-elution data, standalone PrInCE is directly accessed through its own icon. PrInCE can be downloaded for free [13]. Detailed documentation of all the code as well as further instructions for running the software are provided.

\section{Datasets}

This pipeline requires co-fractionation profiles of single proteins, where co-elution is evidence of co-complex membership. Each co-fractionation profile, e.g. a chromatogram, is a row in a .csv file. Co-fractionation profiles are grouped by both experimental condition and replicate number. Separate .csv files are used for different experimental conditions, and the replicate number of each chromatogram is recorded by a column in each file. We provide a test dataset on Github as an example of correct formatting.

\section{Reference database of known complexes}

This pipeline requires a reference database of known protein complexes. A portion of the proteins in these reference complexes must also be quantified in the experimental data, as the reference complexes provide the template by which novel interactions are predicted. We found that manually curated databases that rely on 
a

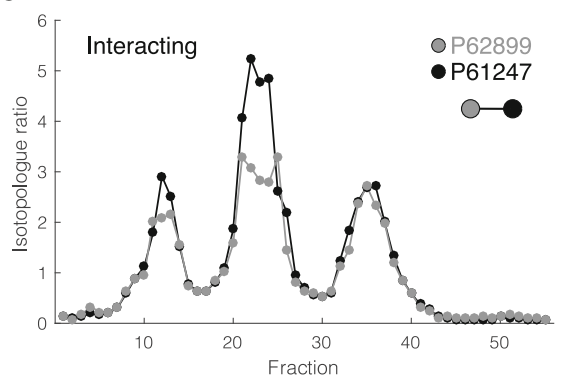

b

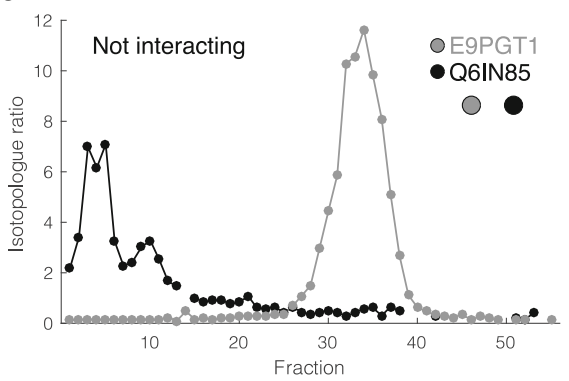

C
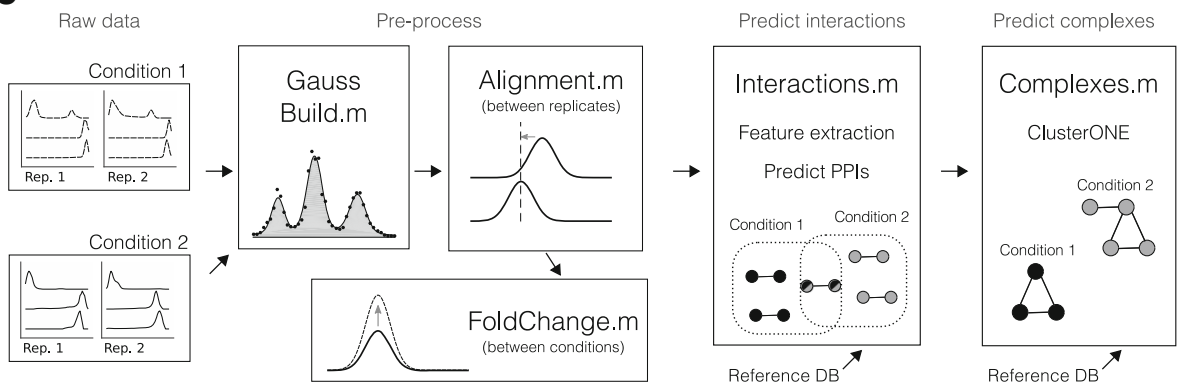

Fig. 1 Pipeline overview. a. Co-fractionation profiles from known interactors, ribosomal proteins P61247 (black) and P62899 (grey). b. Co-fractionation profiles from non- interacting protein pair, Q6IN85 (black) and E9PGT1 (grey). c. Pipeline workflow. Raw data consists of co-fractionation profiles grouped by replicate and condition. In pre-processing, Gaussian mixture models are fit to each co-fractionation profile to obtain peak height, width, and center. If there are multiple replicates, the Alignment module adjusts profiles such that Gaussian peaks for the same protein occur in the same fraction across replicates. Changes in protein amounts between conditions, i.e. fold changes, are computed in the FoldChange module. Inter- actions between pairs of proteins are predicted by first calculating distance measures between each pair of proteins and feeding these into a Naive Bayes supervised learning classifier. Known (non-)interactions from a reference database, e.g. CORUM, are used for training. Finally, the list of predicted pairwise interactions is processed by an optimized ClusterONE algorithm [16] to predict protein complexes

experimental evidence, such as CORUM [14], lead to a high number of predicted interactions.

\section{Pipeline workflow}

\section{Data pre-processing (GaussBuild.M, Alignment.M)}

Module GaussBuild.m uses Gaussian model fitting to identify the location, width, and height of peaks in the co-fractionation data. Any co-fractionation profile with data in at least five fractions is chosen for model fitting. First, single missing values in co-fractionation profiles are imputed as the mean of neighbouring data points. Remaining missing values are imputed as zeros, and cofractionation profiles are smoothed by a sliding average with a width of 5 data points. Five Gaussian mixture models are fit to each profile. These models are mixtures of $1,2,3,4$ or 5 Guassians, respectively. Fitted parameters $A, \mu$, and $\sigma$ are the Gaussian height, center, and width, respectively. In order to reduce the sensitivity to outliers, robust fitting is performed using the $L 1$ norm. For each profile, model selection is performed by selecting minimum AIC values.

Slight differences between the elution time of replicates are corrected by module Alignment.m, using the assumption that proteins with a single, well-defined chromatogram peak should elute in the same fraction in every replicate [11].

\section{Fold changes between conditions (FoldChanges.M)}

Within a single replicate, the protein abundance ratio, i.e. fold change, is calculated between conditions for each protein (FoldChanges.m). If there are multiple replicates, this module also calculates significance using a paired t-test. Fold changes are calculated using data centered on the Gaussian peaks identified by GaussBuild.m [11].

\section{Predicting interactions (Interactions.M)}

Quantifying co-fractionation with distance measures PPI prediction begins by calculating the effective distance between the co-fractionation profiles of every pair of proteins. We use five distance measures to quantify different aspects of co-fractionation profile similarity. For all distance measures, a value close to zero signals high similarity between co-fractionation 
profiles. These five metrics are not exhaustive, but in practice we found there was little value in additional measures. For a pair of co-fractionation profiles $c_{i}, c_{j}$, these distance measures are

- One minus correlation coefficient, $1-R_{\text {corr: }}$ One minus the Pearson correlation coefficient between $c i$ and $c j$.

- Correlation $p$-value, $p_{\text {corr }}$ Corresponding $p$-value to $1-R_{\text {corr }}$

- Euclidean distance between co-fractionation profiles $c i$ and $c j, E$.

- Peak location, $P$ : Calculated as the difference, in fractions, between the locations of the maximum values of $c i$ and $c j$.

- Co-apex score, $C A$ : Euclidean distance between the closest $(\mu, \sigma)$ pairs, where $\mu$ and $\sigma$ are Gaussian parameters fitted to $c i$ and $c_{j}$. For example, if $c_{i}$ is fit by two Gaussians with $(\mu, \sigma)$ equal to $(5,1)$ and $(45$, 3 ), and $c_{j}$ is fit by one Gaussian with parameters $(45,2)$,

$C A=\sqrt{(45-45)^{2}+(3-2)^{2}}=1$. Thus

chromatograms with at least one pair of similar

Gaussian peaks will have a low (similar) Co-apex score.

Predicting interactions via similarity to reference Combined with a reference database such as CORUM, these five distance measures can be used to predict novel PPIs. Our pipeline uses a machine learning classifier to do this $[6,15]$. Specifically, we train a Naïve Bayes classifier, which evaluates how closely the distance measures for a candidate protein-protein pair resemble the distance measures observed for reference interactions. Distance measures are normalized such that their means are 0 and standard deviations 1 . To reject uninformative distance measures, feature selection is performed prior to classification using a Fisher ratio $>2$. The contribution of each feature to prediction performance depends on the dataset, although in general the most-informative (least-rejected) features are 1- $R_{\text {corn }} P$, and CA. Distance measures are combined across replicates (but not conditions) for each protein-protein pair. Class labels are assigned based on the reference database. Reference protein pairs that occur in the same complex are gold standard interactions (interacting or "intra-complex" label). Proteins that are found in the reference database individually but do not occur within the same complex are labeled non-interacting ("inter-complex") and are false positive interactions [6]. Novel interactions are those where one or both members are not in the reference database.

The Naïve Bayes classifier returns the probability that putative protein pairs are interacting. Interaction probabilities are calculated separately for each experimental condition. We use a $k$-fold cross-validation scheme to avoid over-fitting. $k=15$ is used as a tradeoff between computation time and classification accuracy. The classifier calculates an interaction probability for every protein pair. Self-interactions are not considered.

By applying a threshold to interaction probability returned by the classifier, protein pairs are separated into predicted interactions and predicted non-interactions. The probability threshold is chosen so that the resulting interaction list has a desired ratio of true positives (intra-complex) and false positives (inter-complex), quantified as precision $T P /(T P+F P)$, where $T P$ and $F P$ are the number of true positives and false positives. The desired precision is chosen by the user.

Finally, we express the confidence of each predicted interaction by reformulating interaction probability as an interaction score. A predicted interaction's score is equal to the precision of all predicted interactions with an interaction probability greater than or equal to it. Although interaction probability and score are largely equivalent, interaction score has two advantages. First, interaction score is more human readable, since the dynamic range of predicted interaction probabilities is often quite small. Second, the use of interaction score makes it trivial to generate interaction lists with a desired precision.

\section{Predicting complexes (Complexes.M)}

Complexes are predicted from the list of pairwise interactions using the ClusterONE algorithm [16]. The primary benefit of ClusterONE over other algorithms is that ClusterONE can predict the same protein in multiple complexes. Two parameters, $p$ and dens are optimized via grid search to produce the most reference-like complexes. $p$ represents the number of unknown pairwise interactions, and dens is a threshold for the minimum density of a complex, where complex density is defined as the sum of weighted internal edges divided by $N(N-1) / 2$. Parameters are optimized to maximize either the matching ratio [16] or geometric accuracy [17] between predicted and reference complexes. Since there are possibly multiple interaction lists - a list of all predicted interactions as well as lists specific to each experimental condition - complexes can be built for each experimental condition separately, as well as an overall complex set from the aggregate interactome.

\section{Test datasets}

For this study, we tested PrInCE on four co-fractionation datasets, each composed of thousands of co-fractionation profiles (Table 1). D1, D2, and D4 were collected for recently published PCP-SILAC experiments (D1 [18], D2 [11], D4 [8]). D3 is the raw intensity values of the medium 
Table 1 Test dataset summary

\begin{tabular}{|c|c|c|c|c|c|c|}
\hline Dataset & Conditions & Replicates & Fractions & ProteinIDs & $\begin{array}{l}\text { Interactions } \\
(0.50)\end{array}$ & Interactions (0.75) \\
\hline $\mathrm{D} 1^{\mathrm{a}}$ & 2 & 3 & 55 & 3216 & 19,740 & 3416 \\
\hline $\mathrm{D} 2^{\mathrm{b}}$ & 2 & 4 & $45-50$ & 3438 & 7240 & 1447 \\
\hline D3 & 1 & 3 & 55 & 3198 & 5691 & 1160 \\
\hline D4 ${ }^{\mathrm{C}}$ & 2 & 3 & 50 & 3844 & 16,430 & 2072 \\
\hline
\end{tabular}

${ }^{\mathrm{a}}[18],{ }^{\mathrm{b}}[11],{ }^{\mathrm{c}}[8]$

channel of D1, which we included as a surrogate for nonSILAC data, and label-free data more generally.

\section{Gold standard references}

We tested how the choice of gold standard reference affects the interactions predicted by PrInCE. First, we predicted interactions using subsets of CORUM drawn under two different schemes. The first scheme was designed to test the effects of the size of the reference set: a fraction of CORUM complexes were drawn randomly $(10 \%, 20 \%, \ldots, 100 \%$ of complexes) and interactions were predicted from dataset D1. The second scheme was designed to test whether interactions could be predicted consistently for different reference sets. To control the number of PPIs we performed a paired analysis, where we divided CORUM into two halves with equal numbers of gold standard PPIs in the data. These halves have no PPIs in common, and interactions were predicted from both halves using a single replicate of dataset D1. The first scheme was repeated 10 times, and the second Scheme 50 times. Second, we predicted interactions from all four datasets using two additional gold standard references: IntAct [19] and hu.MAP [20].

\section{Validation of PrInCE output}

Using these four datasets, we performed computational validations of PrInCE output. First, we tested whether our metric for ranking predicted interactions (interaction score) is consistent with other known evidence for protein interaction. To do so, we calculated the Spearman correlation coefficient between interaction score and these four other, independent measures of protein interaction: (i) whether protein pairs shared at least one Gene Ontology term within GO slim, a condensed version of the full GO ontology [21, 22]; (ii) the Pearson correlation coefficient of protein abundance across 30 human tissues, as taken from the Human Proteome Map (http://www.humanproteomemap.org/, [23]); (iii) whether protein pairs shared at least one subcellular localization annotation within the Human Protein Atlas Database [24]; and (iv) whether protein pairs shared a structurally resolved domain-domain interface, as identified by the database of threedimensional interacting domains (3did) [25]. This validation was performed on predicted interaction lists with an interaction score of 0.50 or greater.

Second, we investigated whether predicted interactions were enriched over non-interactions for the same four measures (shared GO terms, tissue-dependent proteome abundance correlation, shared subcellular localization terms, and shared structurally resolved interfaces). For these interacting versus non-interacting enrichment analyses, we imposed a $10 \%$ breadth cutoff on all annotation terms, such that only annotation terms common to less than $10 \%$ of all proteins in the sample were used. As in [26], we also used the Jaccard index between protein pairs to quantify the extent of shared annotation terms across the entire Gene Ontology. This validation was performed on more stringent interaction lists (interaction score 0.75 or greater).

Third, we re-estimated the precision of our predicted interaction lists using an independent, previously described method [27]. Our definition of false positives as "inter-complex interactions" likely overestimates the number of false positives. To quantify the magnitude of this overestimation, we added random interactions between non-interacting proteins within the reference set to bring the average expression correlation coefficient of all interacting proteins within the reference dataset to the same level as in the predicted interactome under investigation. To avoid training and testing on the same reference interactions, we randomly withheld $1 / 3$ of CORUM complexes as a validation set, and used the remaining $2 / 3$ as a training set to train the Naive Bayes classifier and predict interactions. The average Pearson correlation coefficient in tissue proteome abundance was calculated for the resulting predicted interactions, and it was compared to interactions from the $1 / 3$ of CORUM withheld for testing. We bootstrapped this procedure 100 times to re-estimate the precision of the protein interaction network.

Finally, following the network analysis of [26], we explored the topological properties of the predicted subgraphs by sequentially removing interactions under one of three schemes: (i) highest interaction score first, (ii) lowest interaction score first, or (iii) randomly. This analysis tests whether the interaction network consists of cores of tightly connected proteins linked by weaker or 
more spurious connections. If this is the case, removing weakest interactions first will fragment the network, increasing the number of unconnected subgraphs and lowering their average size, whereas removing the highest scoring interactions first will not fragment the network.

\section{Results}

PrInCE uses a machine learning approach to predict conditional interactomes from co-fractionation data. Four datasets were used to benchmark PrInCE versus a previous pipeline [11], which showed that PRInCE can discover twice the number of predicted PPIs (Fig. 2a) in less than one tenth the time (Fig. 2b). This improved runtime also includes the complexbuilding module, Complexes. $m$, that was not present in the previous version.

\section{Predicting PPIs (Interactions.M)}

Predicting protein-protein interactions (PPIs) is one of the primary functions of this pipeline. Figure 3 illustrates this process using a subset of D1 that contains ribosomal and proteasomal proteins. Each potential interaction, i.e. protein pair, is first identified as either a reference interaction (white), reference non-interaction, i.e. proteins in the reference that do not interact (black), or unknown (grey; Fig. 3a). To score each potential interaction, the similarity of each pair of co-fractionation profiles is then quantified using the five distance measures (Additional file 1: Figure S1; see Methods for definitions). Using these as input to the machine learning classifier, an interaction probability for each protein pair is then calculated, expressing how well each protein pair resembles the collection of reference PPIs (Fig. 3b).

By applying a threshold to interaction probabilities outputted by the classifier, a final interaction list can be generated at a precision specified by the user. For example, a more stringent list containing an estimated $75 \%$ true positives (white), or a more inclusive list with an estimated $50 \%$ true positives (cyan; Fig. 3c). In general, there is a tradeoff between quantity and quality when predicting PPIs, meaning that more PPIs can be predicted at the cost of lowering the precision (Fig. 3d).

How does the number of quantified proteins affect the number of predicted interactions? To investigate, we analyzed random subsets of each dataset. Although there was considerable variability between datasets, in general there is an $N^{2}$ relationship between the number of proteins used as input to PrInCE and the number of interactions returned as output (Additional file 1: Figure S2). For all datasets, fewer than 500 quantified proteins resulted in less than 1000 interaction at $50 \%$ precision. It is important to note that while PrInCE is designed to predict reference-like PPIs, it would be useless if it didn't

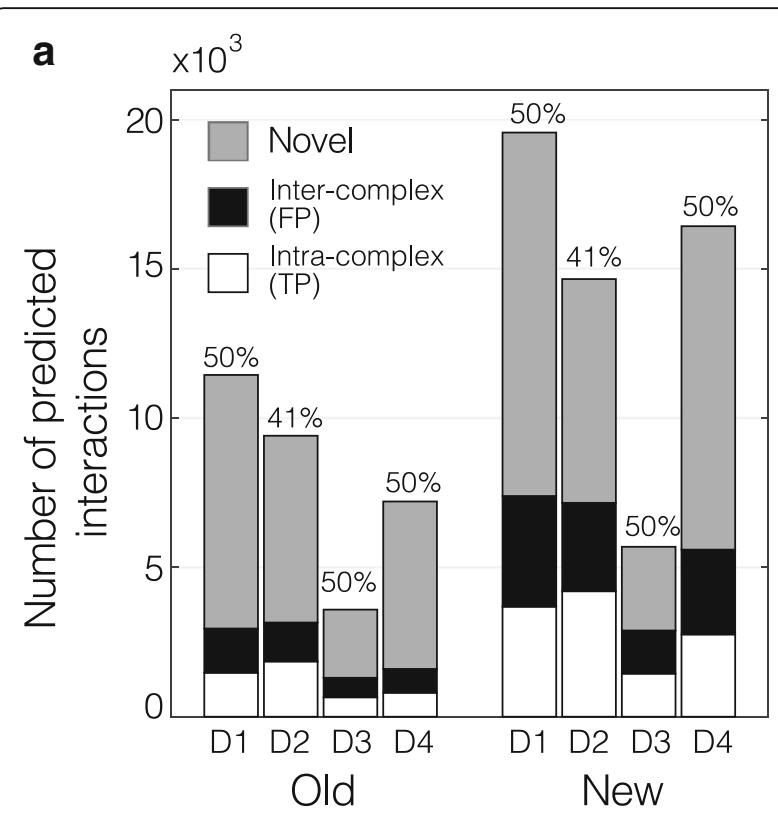

b

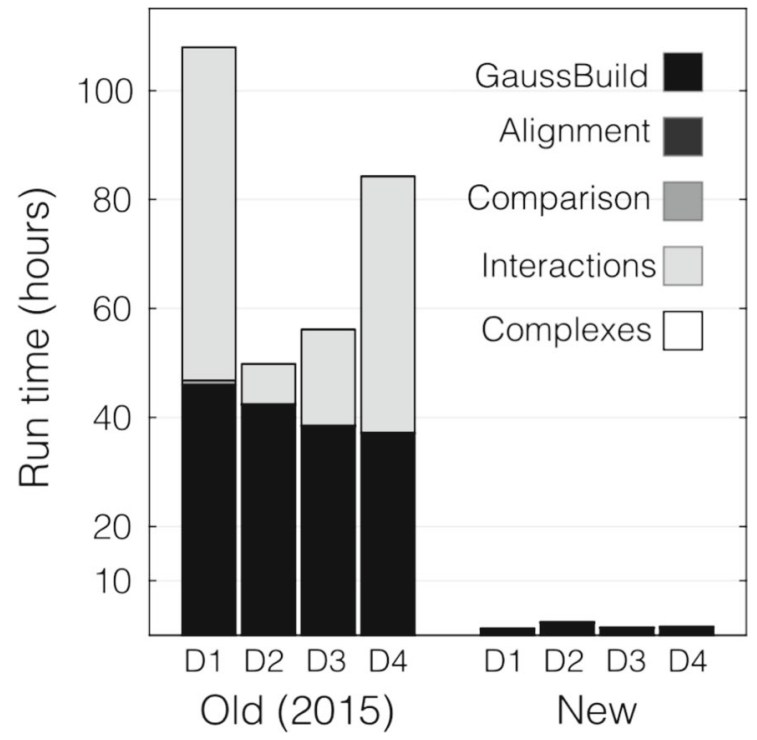

Fig. 2 Improvements to predictive power and run time. a. Number of interactions predicted at 50\% (D1, D3, D4) or 41\% precision (D2). For previously published datasets (D1, D2, D4), precision values and interaction numbers reflect published interaction lists ("Old").

Precision values for "New" output, i.e. from the current pipeline, were chosen to match the Old precision values. CORUM version 2012 was used as a gold standard reference. $\mathbf{b}$. Run time for all modules on a non-performance PC using either the previously published version ("Old (2015)", [11]) or the current version ("New")

also predict novel interactions. That is, PrInCE must predict interactions that are not simply contained in the reference database. Indeed, for the subset of proteins shown in Fig. 3 it can be seen that novel interactions are predicted (Fig. 3c, protein numbers 113 to 237). More broadly, all three datasets we used for benchmarking 

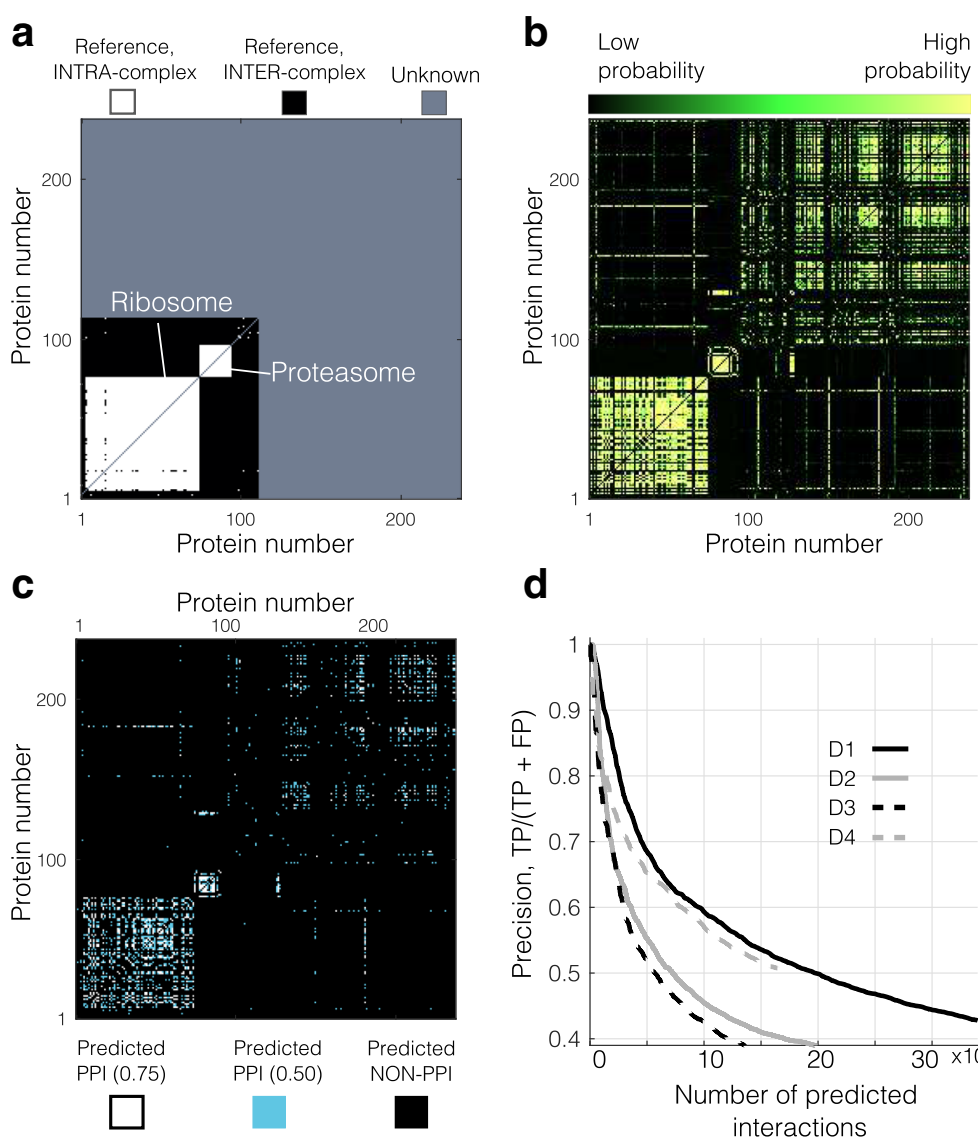

d
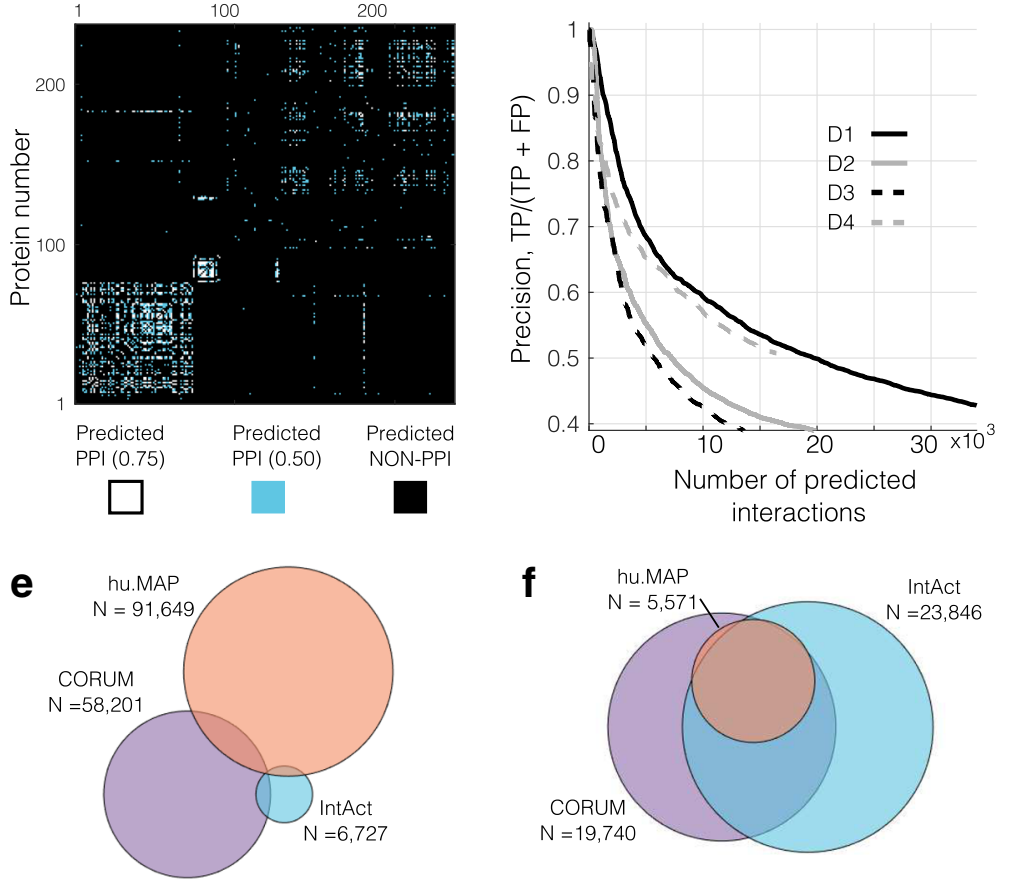

Fig. 3 Predicting interactions (Interactions.m). a. Reference database. Subset of the CORUM reference database, including ribosomal and proteasomal proteins, expressed as a square pairwise matrix. Intra-complex interactions (white) are pairs of proteins from the same reference complex, inter-complex interactions (black) are pairs of proteins contained in the reference that are not co-complex members, and unknown/novel pairs (grey) have one or more protein not contained in the reference. Proteins are sorted according to their peak location. b. Interaction probability for each pair of proteins using the labels in (a) and distance measures. c. Square pairwise matrix of predicted interactions at two precision levels, 50\% (0.50) and 75\% (0.75). Interactions are predicted by applying a constant threshold to interaction score. $\mathbf{d}$. Precision versus accumulated number of interactions. e. Overlap between three gold standard references (CORUM, IntAct, and hu.MAP). f. Predicted interactions using gold standard references from (e). 5527 interactions were commonly predicted from all three gold standards (intersection)

had thousands of novel PPIs predicted at 50\% precision and hundreds to thousands of PPIs at $75 \%$ precision (Fig. 2a, Table 1). In particular, at 50\% precision 16,019 interactions were predicted from D1 that are not contained in the reference.

PrInCE uses a supervised learning algorithm to predict protein-protein interactions (PPIs), meaning it requires examples of both interacting and non- interacting proteins, i.e. a gold standard reference of protein complexes. We sought to investigate how characteristics of the reference impact the interactions predicted by PrInCE. Using subsets of CORUM to simulate the effects of a smaller reference, we see that the number of predicted interactions can vary widely when using relatively small references (Additional file 1 : Figure S3A, B). This is likely due to misestimation of 
the precision of predicted interactions owing to increased effects of noise for smaller references, with spuriously high precision values leading to erroneously large numbers of predicted interactions. However, the predicted interactions that differ between these predicted interactomes tend to be lower scoring, with the highest scoring interactions predicted regardless of the reference (Additional file 1: Figure S3c). Further, entirely non-overlapping CORUM reference sets (Additional file 1: Figure S3D) lead to predicted interactions with $>94 \%$ overlap, on average (average Jaccard index $=0.943+/-0.2$ st.d. between interaction lists predicted from entirely non-overlapping halves of CORUM; Additional file 1: Figure S3E). Therefore, for a given MS/MS dataset, PrInCE tends to predict the same, higher scoring interactions regardless of the reference, although small references can lead to errors in the number of predicted interactions. For large enough references, PrInCE predicts a stable set of interactions, even when gold standard references are incomplete.

Second, we compared the performance of PrInCE trained on CORUM to PrInCE trained on two other gold standards: IntAct, a manually curated database of 1855 protein complexes [19], and hu.MAP, a database synthesized from three high throughput datasets totaling over 9000 mass spectrometry experiments [20]. Although these three gold standards are largely independent, with few common PPIs (average pairwise Jaccard index $=0.03$; Fig. 3e), they lead to predicted interactions with a greater degree of overlap (average pairwise Jaccard index $=0.30$; Fig. 3f; Additional file 1: Table S1). Across all four datasets, there is a pattern for CORUM and IntAct to predict more interactions than hu.MAP (Additional file 1: Figure S4A-C), possibly because CORUM and IntAct are hand-curated. Indeed, gold standard chromatogram pairs given by CORUM and IntAct are more correlated than chromatogram pairs given by hu.MAP, suggesting that hu.MAP contains more false positives (Additional file 1: Figure S4D). However, the larger number of interactions predicted by IntAct may also be an artifact produced by IntAct's relatively small size (130 human complexes) (Additional file 1: Figure S3A). Over all datasets, we find that interactions predicted from multiple gold standards are higher scoring (average interaction score $=0.72$ ) than interactions only predicted using a single gold standard (average score $=0.62$ ). Similarly to our analysis of CORUM subsets, this suggests a stable set of higher-scoring interactions are predicted regardless of the choice of reference (e.g. Fig. 3f).

\section{Predicting protein complexes (Complexes.M)}

Building on predicted PPIs, the second major output of PrInCE is protein complexes. Because buffer conditions in PCP-SILAC are relatively gentle on protein complexes, this module potentially identifies complexes that are unlikely to be identified by immunoprecipitation techniques. To do so, PPIs predicted by Interactions.m are weighted by their interaction score and input into the ClusterONE algorithm [16] to cluster individual PPIs into complexes.

Sorting co-fractionation profiles by their peak location (Fig. 4a) reveals the tendency for groups of proteins to co-elute (Fig. 4b). After analysis with PrInCE, some groups are predicted to be co-complex members. Figure $4 \mathrm{c}$ shows an example protein complex predicted by Complexes.m. The predicted complex (orange and purple) largely overlaps with the $20 \mathrm{~S}$ proteasome contained in the CORUM reference database (black and purple). One member (P28065, orange) was predicted to be participating in the complex. Notably, while P28065 is not in the CORUM database, it is annotated as a proteasomal protein. Thus, using co-elution as the only source of evidence, PrInCE predicted this known cocomplex member of the $20 \mathrm{~S}$ proteasome even though it was missing from the reference.

PrInCE is also capable of predicting entirely novel protein complexes. For example, a four member complex was predicted in dataset D1, of which no proteins were in CORUM (Fig. 4d). Reassuringly, these four proteins (P61923, P53621, P48444, O14579) are all subunits of the coatomer protein complex, a known complex that, while not present in the CORUM database, has substantial low throughput [28-30] and high throughput evidence $[6,8,15]$ supporting its existence. For all complexes predicted by the pipeline (e.g. Fig. 4e; D1, 71 complexes, median size 14), each complex predicted by ClusterONE is matched to a reference complex when possible. Of the 71 protein complexes predicted for D1, 20 were entirely novel, i.e. had no matching reference complex. In general, PrInCE predicts both entirely novel protein complexes and those that recover existing complexes while predicting novel members. The four datasets analyzed in this study produced a total of 291 protein complexes, of which 169 were at least partially matched to a CORUM complex. On average, 31\% of complex subunits were recovered from known complexes while the remaining were novel subunits (Fig. 4f).

\section{Validation of predicted interactions and complexes}

No method for determining protein interactions is perfect, and higher-throughput methods tend to recover noise along with biologically meaningful signal. We estimate how much noise is in the final interaction list by comparing it to a reference of known interactions, e.g. CORUM, and quantifying the signal to noise ratio in terms of precision, i.e. $T P /(T P+F P)$. In order to validate that we are separating signal from noise in a biologically meaningful way, we sought to establish the biological significance of interaction lists generated by PRInCE using independent evidence. First, we wanted to confirm 


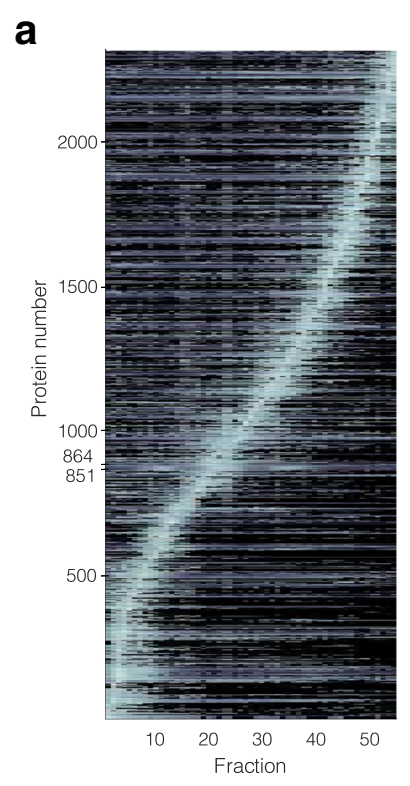

C
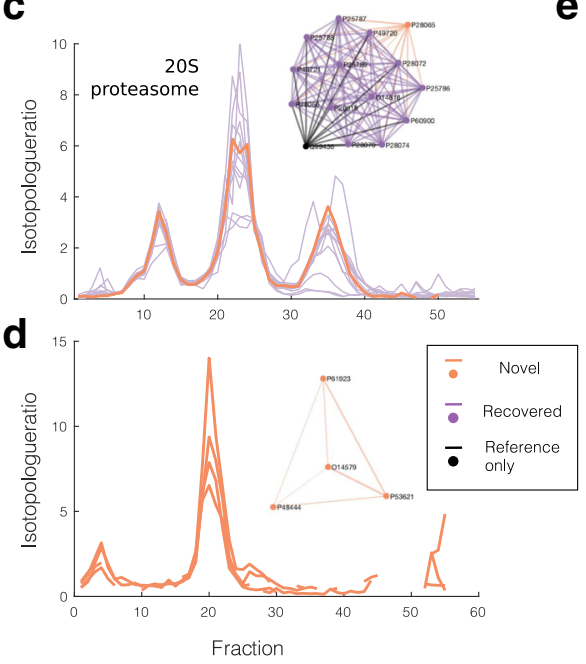

e

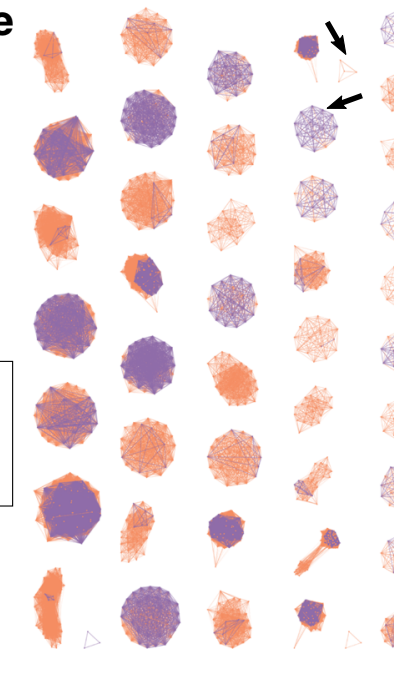

$50 \%$ precision

19,740 interactions

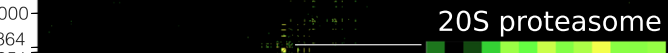

proteasome

500

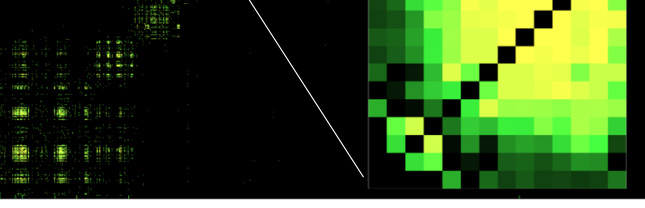

500

$1000 \quad 1500$

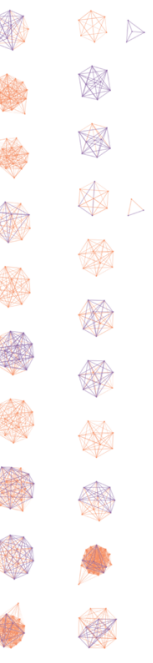

f

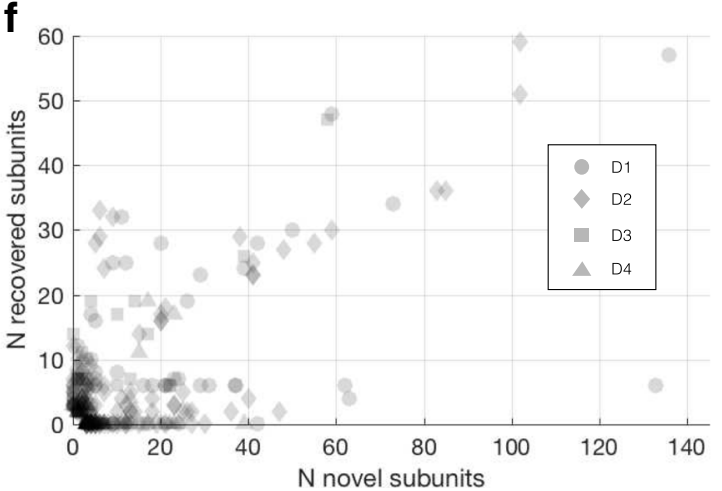

Fig. 4 (See legend on next page.) 
(See figure on previous page.)

Fig. 4 Predicting complexes (Complexes.m). a. 2311 co-fractionation profiles from a single replicate of D1, sorted by peak location. Fourteen $20 \mathrm{~S}$ proteasomal proteins group together (protein numbers 851-864). b. Square connection matrix for same proteins as (a). Colour shows interaction score for all 19,740 interactions with score greater than 0.50. Inset: Close up of the $14 \times 14$ connection matrix for 205 proteasomal members plus other proteins (protein numbers 851-865). c. Co-fractionation profiles for the 14 proteins from B inset, which also correspond to a predicted complex. Profiles of complex members (left) all have a similar shape. When compared to its closest match in CORUM, the 20S proteasome, this predicted complex had 13 overlapping proteins (purple), as well as one protein in the predicted complex that was not in the 20S proteasome (orange). Additionally, there was a single protein from the 205 proteasome that was not in the predicted complex (black). d. Example predicted complex with no match in the CORUM database. e. Force diagrams for all 71 predicted complexes from 19,740 interactions in D1. Same colouring scheme as (d and e). Proteins in known complexes that were not predicted (i.e. Reference-only, black) are omitted for clarity. f. Predicted complexes are composed of known ("recovered") subunits and novel subunits. Data is from all four datasets. The size of each predicted complex is the sum of novel and recovered members

a

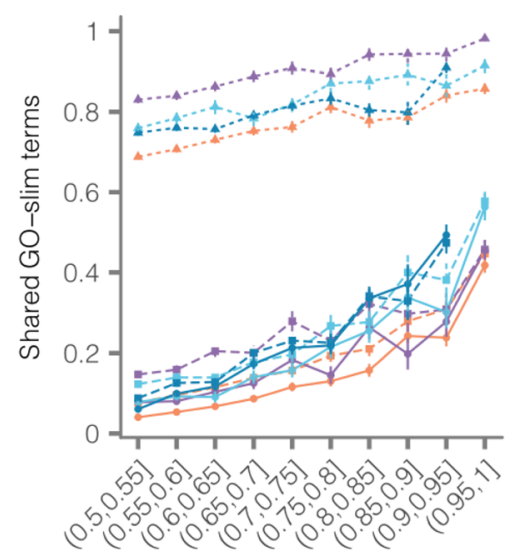

Precision

\section{GO category}

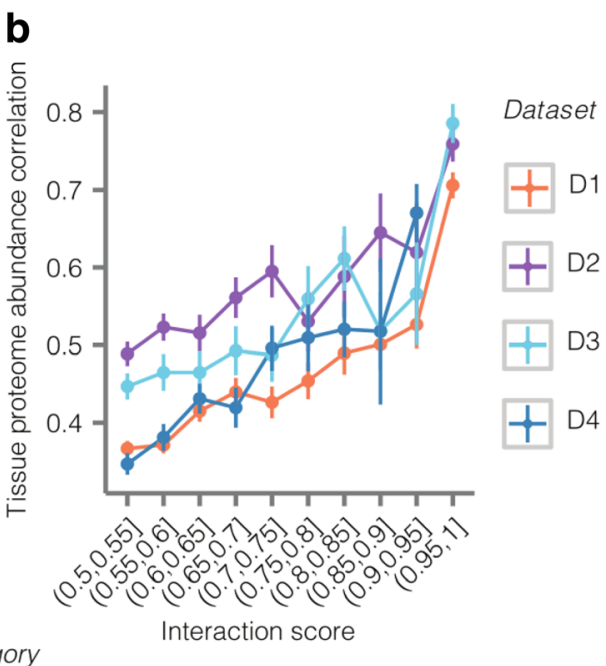

Interaction score

十 Biological process -2 Cellular compartment

C

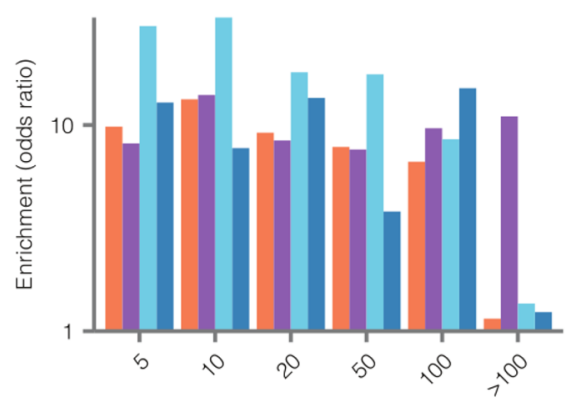

GO term breadth

All categories

Cellular compartment d

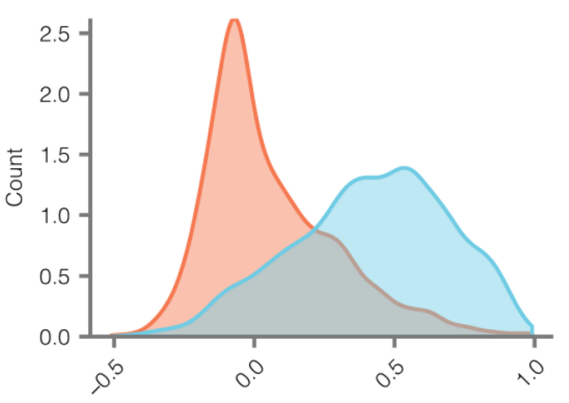

Tissue proteome abundance correlation (PCC)

Not interacting Interacting

Fig. 5 Predicted interactions are enriched for biologically significant attributes, and the degree of enrichment reflects interaction score. a. Fraction of interacting proteins with at least one shared GO-slim term as a function of interaction score and ontological domain. Triangle: biological process. Square: cellular component. Circle: molecular function. b. Tissue proteome abundance [23] correlation (Pearson correlation coefficient) as a function of interaction score. c. Interacting proteins in the apoptosis dataset are enriched for shared GO-slim terms relative to non-interacting protein pairs at diverse GO term breadths. $\mathbf{d}$. Distribution of tissue proteome abundance correlations (Pearson correlation coefficients) for interacting and non-interacting protein pairs in D1 
that the measure we use to rank the confidence of predicted interactions, interaction score, is a useful way to identify which interactions are more likely to be true positives. To do so, we tested whether proteins in high score PPIs are more likely to share annotation terms than low score interactions. Indeed, for every GO-slim annotation category, as interaction score increased, so did the proportion of interactions sharing at least one annotation term (Fig. 5a, Additional file 1: Table S2). Similarly, interacting protein pairs were more likely to be coexpressed across human tissues (Pearson correlation coefficient $\geq 0.75$ ) (Fig. 5b), share at least one subcellular localization term (Additional file 1: Figure S5A), and have a structurally resolved domain-domain interaction (Additional file 1: Figure S5B). Therefore, the ranking system used by this pipeline is biologically meaningful, as demonstrated by independent sources of evidence.

How do predicted interactions differ from predicted non-interactions? A well-performing pipeline returns predicted classes that are, at least by some measures, cleanly separated. To assess this, we first compared Jaccard indices [26], which measure the degree to which protein pairs share annotation terms, between noninteracting protein pairs (cyan), medium-confidence predictions (orange), and high-confidence (purple; Additional file 1: Figures. S5C, S6A-C). Compared to non-interacting proteins, high-confidence interactions show a bias towards larger Jaccard indices, as do mediumconfidence interactions, although to a lesser degree.

We next used enrichment values to quantify the tendency for predicted interacting proteins to share annotation terms. In general, interacting proteins were about $10 \times$ more likely to share $\mathrm{GO}$ annotation terms than non-interacting proteins (Fig. 5c, Additional file 1: Figure S6D-F). Moreover, enrichment was relatively independent of the breadth of the annotation terms, where breadth describes the number of annotated proteins per annotation term [31]. We found that interacting proteins were significantly enriched for nearly all validation measures used here (Table 2). Finally, comparing how well tissue-dependent protein abundance correlates between protein pairs [23] shows that protein abundance is more correlated between predicted interacting protein pairs versus predicted non-interactions (Fig. 5d, Additional file 1: Figure S6G-J). Therefore, predicted interactions returned by PrInCE are more enriched than predicted non-interactions for external evidence of interacting. Importantly, this external evidence is independent of the evidence used within the pipeline. The same analysis was repeated to compare interactions predicted by PrInCE to previously published interaction lists $[8,11]$. To do so, we matched the number of interactions in the published lists by taking that number of top-ranked interactions predicted by PrInCE. In 15 out 18 comparisons of enrichment values, interactions predicted by PrInCE were measured to be more enriched for external evidence of interaction than previously published lists (Additional file 1: Table S3).

Calculating the precision of the interactions predicted by PrInCE is crucial for minimizing the number of false positives. To estimate precision, both the numbers of true and false positives must be calculated. The reference database provides a list of true positive interactions (intra- complex). However, since no comparable database of false positive interactions exists, we make the assumption that pairs of interacting proteins which are both present in the reference, but not reported by the reference to interact, are false positives (inter-complex). Several of these false positives are likely to be true interactions that simply have not been previously discovered and thus not included in the reference, meaning that PrInCE likely underestimates the true precision of the interactions. Using the method outlined in [27] to re-estimate precision, we found that, indeed, the stated precision is a conservative estimate of the confidence of the predicted interaction list (Fig. 6).

Table 2 Interacting versus non-interacting terms for shared annotation terms (GO, Subcellular Localization), tissue-dependent proteome abundance, and shared structurally resolved binding domains

\begin{tabular}{|c|c|c|c|c|c|c|}
\hline \multirow[t]{2}{*}{ Dataset } & GO & GO & GO & Proteome & Subcellular & Structurally \\
\hline & $\mathrm{CC}$ & $\mathrm{BP}$ & MF & Abundance & Localization & Resolved \\
\hline \multirow[t]{2}{*}{ D1 } & 1.2 & 19.6 & 13.6 & 8.7 & 2.7 & 13 \\
\hline & 0.13 & $<1 \mathrm{e}-300$ & $<1 \mathrm{e}-300$ & $<1 \mathrm{e}-300$ & $6 e-21$ & $2 e-275$ \\
\hline \multirow[t]{2}{*}{ D2 } & 1.94 & 12.2 & 10.2 & 7.7 & 3.2 & 14 \\
\hline & $2 e-8$ & $<1 e-300$ & $2 e-266$ & $4 e-264$ & $2 e-8$ & $4 e-267$ \\
\hline \multirow[t]{2}{*}{ D3 } & 2.15 & 16.8 & 13.7 & 12 & 2.5 & 15 \\
\hline & $1 e-4$ & $<1 \mathrm{e}-300$ & $1 e-288$ & $6 e-281$ & $3 e-4$ & $1 e-135$ \\
\hline \multirow[t]{2}{*}{ D4 } & 3.13 & 16.1 & 13.5 & 10 & 2.4 & 11 \\
\hline & 1e-51 & $<1 e-300$ & $<1 \mathrm{e}-300$ & $<1 e-300$ & $2 e-6$ & $<1 \mathrm{e}-300$ \\
\hline
\end{tabular}

Fold values (top numbers) and hypergeometric test $p$-values (bottom numbers). Annotation terms were first filtered using a 10\% breadth cutoff 


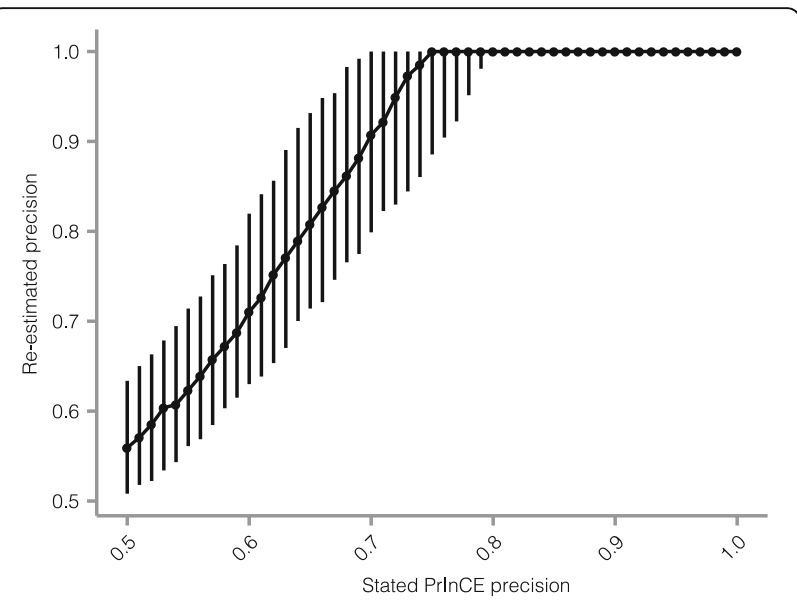

Fig. 6 PrInCE precision of the predicted interaction list is a conservative estimate of the number of false positives. Predicted interaction lists were generated for dataset D1 at multiple user-defined precision levels (PrlnCE precision), and their precision was re-estimated (Mrowka precision [27]). PrInCE lists were generated using a random 2/3 subset of the CORUM reference and precision was re-estimated using the remaining 1/3. Median values from 100 iterations are shown, and bars show the interquartile range

Finally, we explored the topological properties of the predicted network, i.e. how the network is connected. Specifically, as is postulated for other PPI networks returned by high-throughput techniques [26], we validated the hypothesis that predicted networks should consist of small subsets of highly connected proteins, which are more loosely linked to each other by relatively weak connections. This connectivity structure denotes well-defined subgraphs connected by weaker signaling and/or spurious false positive interactions. To analyze the topology, we used an approach described by [26], wherein interactions are removed sequentially from the network: removing the lowest confidence interactions first should fragment the network by revealing islands of isolated subgraphs; removing the highest confidence interactions should lead to no fragmentation. Indeed, removing low confidence interactions first produced a network with a greater number (Additional file 1: Figure S7A, purple) of relatively smaller subgraphs (Additional file 1: Figure S7B), i.e. fragmentation. Removing interactions in this order rapidly fragmented the largest subgraph (Additional file 1: Figure S7C). Removing highconfidence interactions first did not have this effect (Additional file 1: Figure S7, orange). Similar results were obtained for other datasets (Additional file 1: Figure S7E-P).

\section{Discussion}

A machine learning classifier provides improvements over simply sorting protein-protein pairs by how similarly they co-elute, as it provides an automated method for combining multiple measures of co-elution. We chose the Naive Bayes classifier because it is computationally inexpensive and surprisingly powerful given its relative simplicity. Indeed, when comparing the Naive Bayes ("fitcnb", Matlab) to a Support Vector Machine classifier ("fitcsvm", Matlab) we found the Naive Bayes predicted similar or greater numbers of interactions at a given precision level, depending on the dataset (data not shown).

One limitation of our technique is that it requires a suitable gold standard reference of known protein complexes. For mammalian datasets we recommend using the CORUM database, as it is large enough, entirely hand-curated, and accurately describes co-elution data. For yeast or E. coli datasets we recommend the IntAct database. Because false positives are defined as intercomplex pairs that are not also intra-complex pairs, gold standard databases of binary protein pairs, such as STRING, are not suitable. If neither CORUM nor IntAct are suitable, in general we recommend that the reference be large enough $(>10,000$ gold standard protein pairs in the MS/MS dataset, Additional file 1: Figure S3B) and accurately describe the dataset, measured, for example, by high correlation (Pearson $R>0.4$ ) between gold standard co-elution curves (Additional file 1: Figure S4D). Since protein complexes are variable, not all known interactions will occur at any one time or under one set of biological conditions. Therefore, the suitability of a reference database, determined by the fraction of gold standard interactions that were indeed physically interacting in the sample, is crucial. Failure of the gold standard reference to accurately describe the data will result in poor classification performance and, ultimately, a short or empty list of predicted interactions (e.g. hu.MAP, Additional file 1: Figure S4A-C).

Early versions of this pipeline were designed for the analysis of (PCP-) SILAC datasets. A major strength of SILAC experiments is that they allow conditional experiments to be performed simultaneously, minimizing experimental variability between conditions. However, the analysis here of dataset D3, a surrogate for a nonSILAC labelled dataset, demonstrates that PrInCE is not limited to analyzing SILAC data. In fact, PrInCE can analyze any dataset with co-fractionation profiles for single proteins where co-fractionation is meaningful evidence of co-complex membership, and for which there exists a suitable reference.

\section{Conclusions}

PrInCE provides a powerful tool for predicting interactomes from co-fractionation experiments. It greatly simplifies the task of analyzing co-fractionation datasets, requiring at most installation and simple command line tools. Building on preliminary versions of a bioinformatics treatment $[8,11]$, PrInCE predicts nearly twice as many protein interactions at 
the same stringency with a $97 \%$ decrease in run time (Fig. 2). PrInCE also offers increased functionality over previous versions, providing a module for automated, optimized prediction of protein complexes using the ClusterONE algorithm [16]. Importantly, PrInCE is available as a standalone executable program, meaning access to Matlab is not required. Finally, at the same number of interactions, interactions predicted by PrInCE are more supported by external, validating evidence than previous versions, as quantified by a greater enrichment of shared annotation terms (Additional file 1: Table S3).

\section{Additional file}

Additional file 1: Supplementary Figures and Tables. (DOCX 4945 kb)

\section{Abbreviations}

AP-MS: Affinity purification mass spectrometry; PCP: Protein correlation profiling; PPI: Protein-protein interaction; PrInCE: Predicting interactomes from co-elution; SILAC: Stable isotope labelling by amino acids in cell culture; Y2H: Yeast two-hybrid

\section{Acknowledgements}

We thank A. Prudova and A. McAfee for critical suggestions. M.A.S. is supported by a CIHR Frederick Banting and Charles Best Canada Graduate Scholarship, a UBC Four Year Fellowship, and a Vancouver Coastal HealthCIHR-UBC MD/PhD Studentship Award.

\section{Funding}

This work was supported by funding from Genome Canada and Genome British Columbia (project 214PRO) and the Canadian Institutes of Health Research (MOP77688) to L.J.F. Funding bodies played no role in the design or conclusions of this work.

\section{Availability of data and materials}

The three published co-elution datasets analyzed during the current study are available from doi: 10.1038/nmeth.2131, doi: 10.1016/j.jprot.2014.10.024, and doi: $10.15252 / \mathrm{msb} .20167067$. The PrInCE analysis pipeline is available at https://github.com/fosterlab/PrlnCE.

\section{Authors' contributions}

RGS analyzed and interpreted the data, wrote the PrInCE software, and drafted the manuscript and revised it. MS performed the validation analysis and critically revised the manuscript. NS made significant contributions to conception and design of the study and acquired two of the datasets analyzed here. LF made substantial contributions to conception and design of the study and critically revised the manuscript. All authors read and approved the final manuscript.

\section{Ethics approval and consent to participate}

Not applicable.

\section{Consent for publication}

Not applicable.

\section{Competing interests}

The authors declare that they have no competing interests.

\section{Publisher's Note}

Springer Nature remains neutral with regard to jurisdictional claims in published maps and institutional affiliations.

\section{Author details}

'Michael Smith Laboratories, University of British Columbia, Vancouver V6T 1Z4, Canada. ${ }^{2}$ Doherty Institute, University of Melbourne, Melbourne,
Australia. ${ }^{3}$ Department of Biochemistry, University of British Columbia, Vancouver V6T 1Z3, Canada.

Received: 2 June 2017 Accepted: 9 October 2017

Published online: 23 October 2017

References

1. Arifuzzaman M, Maeda M, Itoh A, Nishikata K, Takita C, Saito R, et al. Large-scale identification of protein-protein interaction of Escherichia Coli K-12. Genome Res. 2006;16:686-91.

2. Gavin A-C, Aloy P, Grandi P, Krause R, Boesche M, Marzioch M, et al. Proteome survey reveals modularity of the yeast cell machinery. Nature. 2006:440:631-6.

3. Krogan NJ, Cagney G, Yu H, Zhong G, Guo X, Ignatchenko A, et al. Global landscape of protein complexes in the yeast Saccharomyces Cerevisiae. Nature. 2006:440:637-43.

4. Kühner S, van Noort V, Betts MJ, Leo-Macias A, Batisse C, Rode M, et al. Proteome organization in a genome-reduced bacterium. Science. 2009;326:1235-40.

5. Dunham WH, Mullin M, Gingras A-C. Affinity-purification coupled to mass spectrometry: basic principles and strategies. Proteomics. 2012;12:1576-90.

6. Havugimana PC, Hart GT, Nepusz T, Yang H, Turinsky AL, Li Z, et al. A census of human soluble protein complexes. Cell. 2012;150:1068-81.

7. Heide H, Bleier L, Steger M, Ackermann J, Dröse S, Schwamb B, et al. Complexome profiling identifies TMEM126B as a component of the mitochondrial complex I assembly complex. Cell Metab. 2012;16:538-49.

8. Kristensen AR, Gsponer J, Foster LJA. High-throughput approach for measuring temporal changes in the interactome. Nat Methods. 2012;9:907-9.

9. Brückner A, Polge C, Lentze N, Auerbach D, Schlattner U. Yeast two-hybrid, a powerful tool for systems biology. Int J Mol Sci. 2009;10:2763-88.

10. Choi H, Larsen B, Lin Z-Y, Breitkreutz A, Mellacheruvu D, Fermin D, et al. SAINT: probabilistic scoring of affinity purification-mass spectrometry data. Nat Methods. 2011;8:70-3.

11. Scott NE, Brown LM, Kristensen AR, Foster $\perp$. Development of a computational framework for the analysis of protein correlation profiling and spatial proteomics experiments. J Proteome. 2015;118:112-29.

12. Wan C, Liu J, Fong V, Lugowski A, Stoilova S, Bethune-Waddell D, et al. ComplexQuant: high-throughput computational pipeline for the global quantitative analysis of endogenous soluble protein complexes using high resolution protein HPLC and precision label-free LC/MS/MS. J Proteome. 2013;81:102-11

13. PrInCE: Bioinformatics pipeline for predicting protein interactomes via co-elution. Matlab. Foster lab; 2016. https://github.com/fosterlab/PrlnCE. Accessed 26 May 2017

14. Ruepp A, Waegele B, Lechner M, Brauner B, Dunger-Kaltenbach I, Fobo G, et al. CORUM: the comprehensive resource of mammalian protein complexes-2009. Nucleic Acids Res. 2010;38(suppl 1):D497-501.

15. Wan C, Borgeson B, Phanse S, Tu F, Drew K, Clark G, et al. Panorama of ancient metazoan macromolecular complexes. Nature. 2015:525:339-44.

16. Nepusz T, Yu H, Paccanaro A. Detecting overlapping protein complexes in protein-protein interaction networks. Nat Methods. 2012;9:471-2.

17. Brohee S, Van Helden J. Evaluation of clustering algorithms for proteinprotein interaction networks. BMC Bioinf. 2006;7:488.

18. Scott NE, Rogers LD, Prudova A, Brown NF, Fortelny N, Overall CM, et al. Interactome disassembly during apoptosis occurs independent of caspase cleavage. Mol Syst Biol. 2017;13:906.

19. Meldal BH, Forner-Martinez O, Costanzo MC, Dana J, Demeter J, Dumousseau $M$, et al. The complex portal-an encyclopaedia of macromolecular complexes. Nucleic Acids Res. 2014;43:D479-84.

20. Drew K, Lee C, Huizar RL, Tu F, Borgeson B, McWhite CD, et al. Integration of over 9,000 mass spectrometry experiments builds a global map of human protein complexes. Mol Syst Biol. 2017;13:932.

21. Ashburner M, Ball CA, Blake JA, Botstein D, Butler H, Cherry JM, et al. Gene ontology: tool for the unification of biology. Nat Genet. 2000;25:25-9.

22. Consortium GO. Gene ontology consortium: going forward. Nucleic Acids Res. 2015;43:D1049-56

23. Kim M-S, Pinto SM, Getnet D, Nirujogi RS, Manda SS, Chaerkady R, et al. A draft map of the human proteome. Nature. 2014;509:575-81.

24. Uhlén $M$, Fagerberg $L$, Hallström BM, Lindskog C, Oksvold P, Mardinoglu A, et al. Tissue-based map of the human proteome. Science. 2015;347:1260419. 
25. Mosca R, Céol A, Stein A, Olivella R, Aloy P. 3did: a catalog of domainbased interactions of known three-dimensional structure. Nucleic Acids Res. 2013;42:D374-9.

26. Hein MY, Hubner NC, Poser I, Cox J, Nagaraj N, Toyoda Y, et al. A human interactome in three quantitative dimensions organized by stoichiometries and abundances. Cell. 2015;163:712-23.

27. Mrowka R, Patzak A, Herzel H. Is there a bias in proteome research? Genome Res. 2001;11:1971-3.

28. Eugster A, Frigerio G, Dale M, Duden R. COP I domains required for coatomer integrity, and novel interactions with ARF and ARF-GAP. EMBO J. 2000;19:3905-17.

29. Faulstich D, Auerbach S, Orci L, Ravazzola M, Wegehingel S, Lottspeich F, et al. Architecture of coatomer: molecular characterization of d-COP and protein interactions within the complex. J Cell Biol. 1996;135:53-62.

30. Harter C, Wieland FTA. Single binding site for dilysine retrieval motifs and p23 within the $y$ subunit of coatomer. Proc Natl Acad Sci. 1998;95:11649-54.

31. Simonis N, Rual J-F, Carvunis A-R, Tasan M, Lemmens I, Hirozane-Kishikawa T, et al. Empirically controlled mapping of the Caenorhabditis Elegans proteinprotein interactome network. Nat Methods. 2009;6:47-54.

\section{Submit your next manuscript to BioMed Central} and we will help you at every step:

- We accept pre-submission inquiries

- Our selector tool helps you to find the most relevant journal

- We provide round the clock customer support

- Convenient online submission

- Thorough peer review

- Inclusion in PubMed and all major indexing services

- Maximum visibility for your research

Submit your manuscript at www.biomedcentral.com/submit 